\title{
Discurso y Pensamiento en el Aula Matemática Chilena
}

\section{Discourse and Thought in the Chilean Mathematics Classroom}

\author{
David Preiss \\ Pontificia Universidad Católica de Chile
}

\author{
Antonia Larraín \\ Universidad Alberto Hurtado
}

\author{
Susana Valenzuela \\ Pontificia Universidad Católica de Chile
}

\begin{abstract}
Este estudio tuvo por objetivo explorar la naturaleza de los procesos de pensamiento matemático presentes en el discurso de profesoras y profesores que enseñan en el segundo ciclo de educación básica de escuelas públicas en Chile. Se codificaron 77 videos de clases de profesores participantes en el Sistema de Evaluación Nacional Docente del Gobierno de Chile, en base a una rúbrica que distinguía 4 formas de pensamiento matemático: resolución mecánica de problemas, resolución razonada de problemas, presentación mecánica de la información y presentación razonada de la información. Se realizaron análisis descriptivos y análisis multivariados de varianza. Los resultados evidencian que el pensamiento matemático docente está focalizado en la presentación mecánica de información y la resolución mecánica de problemas. Los profesores que enseñan en $5^{\circ}$ y $6^{\circ}$ grado dedican significativamente menos tiempo a resolución de problemas que los profesores que enseñan en $7^{\circ}$ y $8^{\circ}$ grado. Los resultados son coherentes con evidencia proveniente de otros estudios que muestran la adherencia de los profesores chilenos a una pedagogía intuitiva externalista, centrada en la práctica repetida de procedimientos.
\end{abstract}

Palabras clave: pensamiento público, enseñanza de matemáticas, estudio de video

The goal of this study was to explore the nature of the processes of mathematical thinking evidenced in the discourse of teachers working at Chilean middle-school level public schools. Seventy seven classroom videos filmed as a part of the Chilean Government National Teaching Assessment System were coded according to a rubric that distinguished 4 forms of mathematical thinking: mechanic problem solving, reasoned problem solving, mechanic information presentation, and reasoned information presentation. Descriptive analysis and multivariate analyses of variance were performed. The results evidence that teachers' mathematical thinking is focused on mechanic information presentation and mechanic problem solving. Teachers working in $5^{\text {th }}$ and $6^{\text {th }}$ grade classrooms significantly allocate less time to problem solving than teachers working in $7^{\text {th }}$ and $8^{\text {th }}$ grade classrooms. These results are coherent with previous evidence showing that Chilean teachers adhere to an intuitive pedagogy focused on the repeated practice of procedures.

Keywords: public thinking, teaching of mathematics, video study

El estudio que reportamos exploró el tipo de pensamiento público dominante en la enseñanza de matemáticas en el segundo ciclo de la educación básica en Chile. Por pensamiento público matemático entendemos los procesos de razonamiento matemático realizados por el profesor cuando se dirige a toda la clase en interacción con los estudiantes. El pensamiento público matemático del profesor en el aula es parte de un proceso instruccional deliberado: es ejecutado

David Preiss, Escuela de Psicología, Pontificia Universidad Católica de Chile, Santiago, Chile; Antonia Larraín, Facultad de Psicología, Universidad Alberto Hurtado, Santiago, Chile; Susana Valenzuela, Centro de Medición MIDE UC, Escuela de Psicología, Pontificia Universidad Católica de Chile, Santiago, Chile.

Investigación financiada por el Centro de Estudios de Políticas y Prácticas en Educación (Proyecto CIE01-CONICYT) y por el Proyecto FONDECYT N 11060389.

Agradecemos el apoyo del Centro de Medición MIDE UC y del Centro de Perfeccionamiento, Experimentación e Investigaciones Pedagógicas, quienes dieron acceso al material incluido en el estudio.

La correspondencia relativa a este artículo debe ser dirigida a Antonia Larraín, Universidad Alberto Hurtado, Almirante Barroso 26, Santiago, Chile. E-mail: alarrain@uahurtado.cl 
por el profesor para socializar a sus estudiantes en las matemáticas, compartiendo con ellos su forma de razonar en el contexto de una clase expositiva.

En el mundo educativo hay cierta tendencia a criticar la clase expositiva, dado que en esta el profesor tiene el control del desarrollo de los contenidos e ideas. Efectivamente, las teorías educacionales contemporáneas promueven un rol más protagónico de los estudiantes (Lemke, 1990; Olson, 2003; Rogoff, Matusov \& White, 1996). La idea de que el estudiante es activo en la construcción de conocimiento se ha interpretado como estar permanentemente en actividad, preguntando, trabajando con sus compañeros o con dispositivos tecnológicos. Sin embargo, la actividad que importa pedagógicamente tiene que ver con la actividad del pensamiento y no necesariamente con la conducta observada. En este sentido, escuchando una buena clase expositiva el estudiante también es activo.

Nuestro interés fue estudiar los procesos de pensamiento público propios del aula de matemática de segundo ciclo básico en Chile. Situamos nuestro estudio en la extensa línea de investigación psicológica educacional sobre discurso docente, con especial atención a las iniciativas recientemente avanzadas en Chile (Milicic, Rosas, Scharager, García \& Godoy, 2008; Preiss, 2009, 2010; Radovic \& Preiss, 2010). Luego, con el propósito de avanzar en el marco conceptual del estudio, discutimos diversas teorías que indican que los procesos de discurso público en la sala son procesos de pensamiento matemático y especificamos aquellos procesos de pensamiento público que favorecen el aprendizaje disciplinar. Al concluir, discutimos —en el contexto de los resultados obtenidos - la creencia de que la clase expositiva, en sí misma, va en desmedro del aprendizaje. Finalmente, situamos estos resultados en el contexto de la evidencia acumulada sobre las fortalezas y debilidades de la práctica docente en Chile.

\section{Discurso en Aula y Aprendizaje}

Existe una larga tradición de investigación que ha tratado de entender la magnitud y características del discurso docente. En Estados Unidos los primeros estudios de interacción en la sala de clases ya reportaban la predominancia del discurso docente en las actividades del aula (Flanders, 1964). En el Reino Unido se reportó que tres cuartos de la actividad de la clase involucraban discurso oral (Galton, Hargreaves, Comber, Wall \& Pell, 1999; Galton, Simon \& Croll, 1980). Sin embargo, esta dominancia del discurso por sobre otro tipo de actividades — como el trabajo independiente en silencio, por ejemplo — no se traduce necesariamente en un adecuado proceso pedagógico. Varios autores reportan, en diferentes contextos escolares, un predominio de formas discursivas con escaso valor para el aprendizaje (Alexander, 2000; Galton et al., 1980; Galton et al., 1999; Nystrand, Gamoran, Kachur \& Prendergast, 1997; Preiss, 2009; Radovic \& Preiss, 2010; Stigler \& Hiebert, 1999).

El patrón de centralidad del profesor es, eventualmente, modificable. Por ejemplo, como resultado de políticas públicas de incentivo al aumento de interacción, se observa en el Reino Unido un cambio hacia un formato de clases más interactivo (Galton et al., 1999). Sin embargo, el proceso de enseñanza sigue teniendo limitaciones, dado que no se dan espacios para que los estudiantes desarrollen sus ideas (Alexander, 2004). En la misma línea, el discurso que prevalece en las actividades en grupos chicos tiene limitaciones adicionales, ya que un porcentaje no menor de tiempo es dedicado a actividades no relacionadas con la tarea y, cuando se trabaja en lo asignado, el intercambio y discusión de ideas es mínimo (Galton \& Williamson, 1992).

En Chile, a partir de la evidencia audiovisual del Sistema de Evaluación del Desempeño Profesional Docente, Docentemás (DM), se ha desarrollado una serie de estudios de video sobre la enseñanza de las matemáticas en la escuela municipal (Araya \& Dartnell, 2009; Preiss et al., en prensa; Radovic \& Preiss, 2010). Preiss (2010) reporta el predominio del docente en el discurso de la sala de clases. Adicionalmente, los resultados sugieren que el discurso docente está focalizado en procesos de ensayo y recuerdo mecánico: las clases tienden a estar organizadas alrededor de la práctica repetida de problemas matemáticos. Radovic y Preiss (2010) constatan que las preguntas de los profesores son generalmente cerradas y los seguimientos son de bajo potencial meta-cognitivo. Preguntas que promueven el habla acerca de los procedimientos ma- 
temáticos son escasas. Tanto Araya y Dartnell (2009) como Radovic y Preiss (2010) muestran que los estudiantes hacen una cantidad menor de preguntas. Araya y Dartnell (2009) observan que los profesores raramente incluyen demostraciones acerca de los contenidos matemáticos trabajados ni ningún rastro de razonamiento deductivo. En una línea complementaria de investigación, Varas, Cubillos y Jiménez (2009) registraron en video clases de matemáticas de $7^{\circ}$ básico (21 profesores) trabajando en el teorema de Pitágoras, mostrando una escasa presencia de procesos públicos de fomento del razonamiento matemático.

\section{El Rol de la Resolución de Problemas en el Discurso Matemático}

La enseñanza del pensamiento matemático es parte de los procesos genéricos por medio de los cuales el proceso de escolarización desarrolla la literacidad (Olson, 2005, 2009). Este proceso de socialización conlleva la inmersión del niño en un mundo donde el uso de diversos sistemas de signos - la notación matemática, en el caso que aquí nos ocupa- expande su habilidad para pensar. Aprender matemáticas es participar de un proceso de socialización, iniciado en la escuela, en los modos de conocer usados por la comunidad de matemáticos y profesores de matemáticas. De acuerdo a Nunes (1999), el aprendizaje de las matemáticas en la escuela consiste en un proceso de re-descripción representacional, en el que los significados que se derivan de esquemas de acción asociados a procesos matemáticos básicos, tales como contar, se asocian a nuevos sistemas de signos que son aprendidos en la sala de clases. Estos procesos de re-descripción - del esquema de acción al signo - son sociales en virtud de la naturaleza convencional de las matemáticas y su anclaje en procesos de interacción social (Nunes, 1999). El trabajo de los profesores consiste, entonces, en favorecer este proceso de re-descripción, impulsando la conexión entre las matemáticas intuitivas, que los estudiantes traen desde fuera de la escuela, y el manejo formal enseñado en el aula. La pregunta fundamental es ¿cómo los profesores pueden promover este proceso?

Si el interés es que el niño comprenda las matemáticas y aprenda no solo "adquiriendo" sino elaborando los procesos y estructuras matemáticas, aquello que debe ser investigado son los procesos de comunicación en los que el pensamiento matemático ocurre (Kieran, Forman \& Sfard, 2001). En efecto, el aprendizaje de las matemáticas es un proceso a través del cual el estudiante se inicia en una forma específica de comunicación, conocida como discurso matemático. Lo primero que debe ocurrir para que haya comprensión de una idea es hablar de esta, es decir, estar expuesto al uso del discurso matemático y tener la posibilidad de hablarlo (Sfard, 2001). Como los procesos de discurso requieren ser entendidos en interacción, los procesos de pensamiento como discurso en la sala de clases no cuentan como un pensamiento del profesor + el pensamiento del estudiante A + el pensamiento del estudiante B, etc., sino como pensamiento colaborativo que ocurre públicamente.

Existe un relativo consenso entre los educadores de matemáticas en que un tipo de actividad matemática que promueve el uso del discurso de la disciplina por parte de los estudiantes y, por tanto, su aprendizaje, es la resolución de problemas (Steinberg, Empson \& Carpenter, 2004). Aunque un enfoque de resolución de problemas no garantiza el desarrollo de pensamiento del estudiante ni la tendencia de un profesor a estimularlo, este involucra formas discursivas que, en sí mismas, promueven que el estudiante piense matemáticamente y no solo ejecute procedimientos rutinarios (Steinberg et al., 2004). Si bien la definición de lo que se entiende por resolución de problemas en la enseñanza de matemáticas es controversial, los expertos tienden a coincidir en que se trata de la búsqueda por parte del estudiante de soluciones a una situación que plantea una incógnita (Chamberlin, 2008). Resolver un problema implica la búsqueda de un procedimiento o método: este no viene dado de antemano ni puede ser aplicado automáticamente.

Entenderemos como problema matemático toda formulación que presenta una incógnita a ser resuelta por procedimientos de cálculo matemático que no son parte evidente de la formulación. Resolución de problemas es entendida como toda actividad matemática cuyo desarrollo requiera que el estudiante explore procedimientos y métodos que no están incorporados de ma- 
nera evidente en la formulación inicial. Por ejemplo, la formulación $2+4=$ ?, aunque plantea una incógnita, puede ser resuelta con la aplicación de métodos rutinarios y automáticos. Esto es radicalmente distinto en el caso de la siguiente formulación: Pedro tendrá 14 años para el mundial de fútbol del año 2014 en Brasil, pero su padre lo llevará a las Olimpíadas del año 2016. ¿Cuántos años tendrá Pedro para las Olimpíadas? Para responder la pregunta y develar la incógnita es necesario aplicar una operación de adición; sin embargo, la operación requiere "traducir" la formulación a lenguaje matemático para luego realizar la operación.

Steinberg et al. (2004) describen cuatro niveles en los que un profesor puede estar involucrado con el pensamiento del niño en la resolución de problemas. Un primer nivel se caracterizaría por la creencia del docente de que los estudiantes no pueden resolver problemas a menos que se les enseñe cómo. Esta creencia estaría presente en aquellos docentes que no proveen oportunidades para que los niños resuelvan los problemas y no les preguntan cómo lo hicieron. Un segundo nivel estaría caracterizado por profesores que creen que los estudiantes pueden resolver problemas sin enseñarles previamente cómo y, aunque hablan del valor de la variedad de métodos para alcanzar una solución, son inconsistentes y poco sistemáticos en las oportunidades que proveen para discutir los diversos métodos. Estos dos primeros niveles no estarían centrados en hacer pensar a los estudiantes, sino en enseñarles procedimientos. Por el contrario, los siguientes dos niveles se caracterizan porque los profesores creen que para que los estudiantes comprendan deben resolver los problemas en sus propios términos. Estos profesores, además de una variedad de oportunidades para resolver problemas autónomamente, proveen espacios para que los estudiantes discutan las soluciones, con el propósito de conocer sus formas de pensar. Estos niveles se diferencian en el detalle en que los profesores son capaces de describir el pensamiento de los estudiantes y en la medida en que este influye en el desarrollo del currículum.

En conclusión, si bien incorporar un enfoque de resolución de problemas parece ser importante para promover el pensamiento matemático en los niños, no es suficiente. Se requiere una actitud reflexiva con respecto a los procesos de pensamiento que el niño utiliza para resolver estos problemas. Más aún, se requiere que el profesor modele formas de hablar propiamente matemáticas acerca de los métodos usados.

\section{Más Allá de Ejecutar: Hablar Para Razonar Matemáticamente}

De acuerdo a Sfard (2001), el aprendizaje de las matemáticas consiste en un proceso de objetivación. Cuando un estudiante es capaz de hablar acerca de una idea matemática usando flexiblemente diversos formatos representacionales construye un objeto matemático. La única manera de construir un objeto matemático, a su vez, es comenzar a hablar de este. Por ejemplo, cuando un estudiante es capaz de hablar de un número en términos equivalentes, como fracciones, números decimales o porcentajes, se entiende que ha construido un objeto matemático: distintos tipos de discurso matemático que han sido independientemente creados se ocupan para hablar de una misma cosa. Para aprender a pensar matemáticamente y construir objetos matemáticos es necesario aprender meta-reglas propias de la disciplina que han sido históricamente construidas y deben ser re-construidas a lo largo del desarrollo de la experiencia matemática del estudiante (Sfard, 2001). En este sentido, no se puede aprender matemáticas sin una guía cuidadosa y sistemática por parte de un adulto, pues los estudiantes solo pueden aprender estas meta-reglas a través de la interacción con un participante experto. No es que el conocimiento matemático se tenga o no; lo que no debe faltar son interacciones discursivas oportunas y efectivas que provean la oportunidad de un intento de comprensión sostenida en el discurso.

Según Sfard (2001), para que el pensamiento público matemático promueva procesos de objetivación es necesario que este mantenga el pensamiento conjunto de profesores y estudiantes, o entre estudiantes, en un mismo foco atencional. No se trata de atender perceptualmente al mismo objeto (por ejemplo, los ángulos de una figura geométrica en la pizarra), sino de pensar esa figura de una manera conjunta. Para mantener el foco atencional de los estudiantes el profesor debe hablar de cómo concibe el objeto atendido, explicitando procedimientos y 
métodos. El profesor puede explicitar la relación del objeto atendido (por ejemplo, ángulos) con otras ideas matemáticas (por ejemplo, área o perímetro), dar cuenta de cómo se origina o se sostiene esa idea, defender una idea con otras piezas de conocimiento matemático, etc. En otras palabras, para enseñar a pensar matemáticamente no es suficiente "presentar" un objeto al que todos pueden atender; es necesario sostener un proceso de significación conjunta, hablando de forma acorde al pensamiento de los estudiantes. Este primer aspecto depende estrechamente de un segundo proceso meta-discursivo: el contexto interaccional del intercambio pedagógico (aun entre dos compañeros). El esfuerzo por explicitar procedimientos, además de depender del conocimiento propiamente matemático de quien explicita, depende del status de la relación entre los participantes: el interés, la actitud y consideración hacia el otro. Si no se tiene claridad acerca del pensamiento del niño o si este no se considera al momento de hablar matemáticamente acerca de un objeto, difícilmente se conseguirá sostener un foco atencional conjunto.

Para aprender a pensar se tiene que estar expuesto a ciertas formas de hablar (y no a cualquiera), por lo que una clase interactiva no es necesariamente mejor que una lectiva ni la actividad en grupos está por sobre la actividad de clase completa; todo depende del tipo de pensamiento que ocurra en una u otra forma de usar funcionalmente los signos. Varios autores plantean que el discurso en la sala de clases que promueve el aprendizaje es un discurso en el que la perspectiva de los estudiantes se toma en cuenta, en que se les desafía a dar razones y a discutir las ideas y se les anima a pensar acerca de sus opiniones y la de sus compañeros (Alexander, 2008; Mercer \& Littleton, 2007). Con respecto al trabajo en grupos pequeños, estudios experimentales muestran que el tipo de discurso que fomenta el aprendizaje se caracteriza por la discusión de ideas contrarias entre los estudiantes (Asterhan \& Schwarz, 2009), bajo el entendido de que, aunque en todo tipo de discurso ocurren procesos de pensamiento, ciertas formas de usar funcionalmente los signos promueven procesos de revisión del propio pensamiento, mientras que en otras predomina el recuerdo mecánico (Mercer \& Littleton, 2007).

En síntesis, si se trata de estudiar procesos de enseñanza de pensamiento matemático, una vía interesante es atender al discurso público. Cuando el profesor y estudiantes hablan dirigiéndose a toda la clase, no se trata solo de hacer públicamente ejercicios, sino de hablar de estos, es decir, pensarlos. Matemáticamente esto equivale o bien a demostrar la idea matemática, argumentarla vinculándola con otras ideas, o explicitar una multiplicidad de formas para realizar un procedimiento. Por consiguiente, en el estudio de la enseñanza de pensamiento matemático la indagación en el tipo de habla acerca de las ideas matemáticas es fundamental. Tal como ha sido sugerido (Chamberlin, 2008; Steinberg et al., 2004), la resolución de problemas es una forma de hablar matemáticamente que promueve la actividad e iniciativa del estudiante, es decir, permite ensayar el uso funcional del signo más allá de lo evidente o automático. De esta manera, la extensión con la que se usa la resolución de problemas en la enseñanza matemática es un eje relevante de atender.

\section{Propósito del Estudio}

El estudio que reportamos tuvo como objeto principal el discurso público en la enseñanza de matemáticas en el segundo ciclo de la educación básica, con el fin de explorar el tipo de pensamiento público matemático. Se atendió al pensamiento conjunto entre estudiantes y profesores según los ejes:

1. Resolución de problemas (RP) versus no resolución de problemas (NRP) y

2. Razonamiento o hablar acerca de un procedimiento o idea matemática (esto es, demostrar, argumentar, vincular distintas operaciones) versus ejecución mecánica de un procedimiento matemático.

Por un lado, entendemos por pensamiento dedicado a la RP todo pensamiento destinado a resolver un desafío matemático que no es presentado preparado para ser resuelto, sino que requiere de procesos de inferencia y ordenamiento de la información por parte del estudiante para llegar al resultado (dar respuesta a la incógnita). El pensamiento NRP incluye todo tipo 
de trabajo matemático que no involucra RP, esto es, ejercicios matemáticos, enseñanza explícita de fórmulas, teoremas, propiedades matemáticas, principios y conceptos.

Por otro lado, por razonamiento nos referimos en este estudio a aquellos procesos de pensamiento en el que estos procesos de razonamiento no ocurren implícitamente, sino que son el foco del habla pública. Para efectos de nuestro estudio, consideramos que existe razonamiento cuando se procede a:

- demostrar una idea, teorema, regla o fórmula, entre otros;

- argumentar a favor o en contra de estas;

- rescatar diferentes formas o métodos.

Por el contrario, consideramos que solo existe ejecución mecánica cuando no se presenta ninguna de estas tres actividades y el discurso se restringe a una coordinación para la ejecución de cálculos (incluyendo la presentación de información necesaria para estos).

Con estos antecedentes, volvimos a revisar algunas de las clases observadas por Preiss (2010) y Radovic y Preiss (2010) con el propósito de complementar sus hallazgos con un estudio sobre el tipo de oportunidades presentes en aquellas clases para desarrollar pensamiento matemático. El objetivo del estudio fue explorar el tipo de pensamiento matemático que ocurre en el discurso público de la enseñanza de matemáticas de segundo ciclo básico en la educación pública en Chile, así como posibles variaciones del mismo según nivel de desempeño docente, curso y edad de los profesores participantes.

\section{Método}

\section{Participantes}

En el año 2005 fueron evaluados por DM 684 profesores de matemáticas de segundo ciclo básico. Del grupo de profesores que consintieron en que su material fuese usado con fines de investigación, 114 profesores fueron seleccionados aleatoriamente por Preiss (2010). De esa muestra, realizamos un nuevo proceso de selección aleatoria de 77 profesores, cuyo material fue re-codificado en este estudio. Al momento de la filmación, 25 de los profesores enseñaban en $5^{\circ}$ básico, 17 en $6^{\circ}, 18$ en $7^{\circ}$ y 15 en $8^{\circ}$. Dos profesores carecían de información del curso en el que enseñaban y cuatro carecían de información respecto de su edad, los que fueron excluidos del análisis multivariado, dado que la edad fue considerada como covariante. Del total de docentes, uno (1,3\%) fue evaluado como insatisfactorio, 15 (19,5\%) fueron evaluados como básicos, 51 como competentes $(66,2 \%)$ y $10(13 \%)$ como destacados.

\section{Material}

Para una descripción del procedimiento de filmación de las clases de DM, ver Preiss (2009). Es importante notar que, por diseño del sistema de evaluación, estas clases deben durar 40 minutos y deben contar con al menos 10 minutos de habla pública. Por lo mismo, estas deben ser consideradas más como una demostración experimental del desempeño usual de los profesores observados que una demostración natural del mismo. Por otro lado, la atención debe estar puesta, en el caso de datos descriptivos, en los porcentajes de tiempo de clase dedicados a la actividad referida por cada código y no en la cantidad absoluta de tiempo dedicada a cada actividad.

\section{Procedimiento}

De los 40 minutos aproximados que duraba cada sesión seleccionamos los episodios en que la actividad involucraba discurso del profesor dirigido a la totalidad del curso (discurso público) y se trabajaban contenidos curriculares. Definimos estos episodios como discurso docente relevante. El resto de los momentos de la clase no fue codificado. 


\section{Codificación del Material}

Todo el discurso docente relevante se codificó como pensamiento dedicado a la RP o como trabajo matemático que no involucraba resolución de problemas (NRP). Paralelamente, se codificaron tanto aquellos episodios RP como NRP según si presentaban o no algún tipo de proceso de razonamiento acerca de las operaciones realizadas. Como proceso de razonamiento entendimos todo tipo de trabajo matemático que implicara procesos de elaboración de la práctica matemática, ya sea a través de demostraciones, procesos de argumentación o contra-argumentación o vinculación entre distintas operaciones o ideas. Cada episodio de pensamiento matemático relevante fue codificado en base a cuatro códigos que conjugan los dos niveles de las dos dimensiones mencionadas:

1. Resolución mecánica de problemas (presencia de RP-ausencia de razonamiento)

2. Resolución razonada de problemas (presencia de RP-presencia de razonamiento)

3. Trabajo matemático mecánico (NRP-ausencia razonamiento)

4. Trabajo matemático razonado (NRP-presencia de razonamiento).

El criterio de segmentación de los episodios fue el cambio de un modo de pensar a otro: cuando se apreciaba el cambio de una categoría a otra se terminaba el episodio y comenzaba el siguiente. En el Anexo hemos ejemplificado los códigos.

Los videos fueron codificados con la ayuda de un software especialmente diseñado para análisis de videos, The Observer (Jansen, Wiertz, Meyer \& Noldus, 2003), el que permite codificar con una alta precisión, minimizando los errores. Fueron codificados por dos codificadores especialmente capacitados. Se doble codificaron 19 videos en dos rondas de ocho videos cada una y una tercera de tres.

Calculamos la confiabilidad en base al porcentaje de acuerdo en cada video, tal como es calculado por The Observer. En la primera ronda el porcentaje de acuerdo entre codificadores superó en todos los casos el 60\%, salvo en dos casos en que se obtuvo 52 y 53\%. Revisamos los desacuerdos e intensificamos la capacitación de los codificadores. Durante la segunda ronda todos los porcentajes de acuerdo superaron el $67 \%$, siendo los siguientes los porcentajes de acuerdo de los ocho videos: $67,68,72,75,78,85$ y 84 . Los videos que fueron doble codificados se recodificaron en conjunto, discutiendo y resolviendo los momentos de desacuerdo. Luego de obtenidos estos buenos niveles de acuerdo, cada codificador codificó independientemente 28 y 30 videos, respectivamente. Durante el proceso de codificación —con el propósito de controlar la mantención de los niveles de acuerdo - se realizó una nueva ronda de doble codificación, obteniendo un porcentaje de acuerdo de 100, 87 y 100, respectivamente, lo que muestra una mantención del nivel de calibración de los codificadores.

\section{Análisis de Datos}

Para evaluar si las variables de interés se veían afectadas por el nivel de desempeño docente y el nivel en que los profesores fueron filmados haciendo clases, realizamos un análisis multivariado de varianza, utilizando el comando GLM en SPSS. Tal como se indicó previamente, cuatro casos que carecían de información sobre su edad fueron omitidos del análisis. Decidimos agrupar en dos niveles la variable evaluación docente, por la disparidad de participantes en cada nivel de la evaluación: agrupamos en un nivel a los profesores cuyo desempeño fue identificado como insatisfactorio o básico (esto es, bajo estándar, $n=14$ ) y en otro nivel a aquellos cuyo desempeño fue identificado como competente o destacado (esto es, sobre estándar, $n=$ 59). La variable curso fue agrupada en dos niveles: $5^{\circ}$ y $6^{\circ}$ básico en un nivel $(n=41)$ y $7^{\circ}$ y $8^{\circ}$ en otro $(n=32)$. Las variables dependientes fueron el tiempo total destinado a los dos tipos dominantes de pensamiento público: trabajo matemático NRP y pensamiento mecánico, ambos considerados en su totalidad. Para controlar las variaciones en el tiempo dedicado a discurso docente relevante y la edad consideramos ambas variables como covariantes. 


\section{Resultados}

\section{Resultados Descriptivos}

El tiempo de pensamiento público relevante para este estudio fue en promedio 1003 segundos $(D E=419)$. De este total, el tiempo dedicado a la RP fue en promedio 317 segundos $(D E=$ 399). Del tiempo de pensamiento público dedicado a la RP, se dedicaba una gran parte del tiempo a la resolución mecánica de problemas $(M=264$ segundos, $D E=334)$, dejando en promedio menos de un minuto para la resolución elaborada de problemas; esto es, el 83\% del tiempo dedicado a resolver problemas se piensa a un nivel rutinario y básico. El trabajo matemático NRP resultó ser el tipo de pensamiento predominante en el discurso docente. En promedio los profesores dedicaban 685 segundos a este $(D E=431)$. Por otro lado, al igual que en el caso del pensamiento dedicado a RP, la mayoría del tiempo dedicado a otro tipo de trabajo matemático se caracterizaba por ser mecánico: 605 segundos $(D E=399)$, mientras solo 81 segundos en promedio se hablaba o razonaba acerca de lo que se pensaba $(D E=136)$. En síntesis, los resultados descriptivos sugieren que el nivel en que se piensan las matemáticas de manera pública en las aulas observadas es predominantemente mecánico. En la minoría de las ocasiones se da un pensamiento que razona acerca de las operaciones o del trabajo matemático realizado.

\section{Más Allá de los Resultados Descriptivos}

En la Tabla 1 presentamos los promedios y desviaciones estándar para cada una de las condiciones del análisis multivariado. Los test multivariados mostraron que el nivel de evaluación docente y el nivel del curso en el que enseña el profesor no tuvieron un impacto significativo, controlando por edad y tiempo de clase relevante. Solo la covariante duración discurso docente relevante fue significativa, $F(2,66)=186,9, p<0,001, \eta_{\mathrm{p}}{ }^{2}=0,85$, a diferencia de la covariante edad, $F(2,66)=1,35, p=0,267$, y los factores Curso, $F(2,66)=2,32, p=0,107$, y Evaluación Docente, $F(2,66)=2,40, p=0,099$.

Los resultados de los test de efectos entre-sujetos fueron los siguientes: mientras la covariante edad no resultó significativa para pensamiento mecánico, $F(1,67)=0,63, p=0,429$, y para NRP, $F(1,67)=2,42, p=0,125$, la covariante duración discurso docente relevante sí resultó significativa para pensamiento mecánico, $F(1,67)=371,02, p<0,001, \eta_{\mathrm{p}}{ }^{2}=0,85, \mathrm{y}$ para NRP, $F(1,67)=33,9, p<0,001, \eta_{\mathrm{p}}{ }^{2}=0,34$. El efecto del Curso sobre el tiempo dedicado a Pensamiento Mecánico no fue significativo, $F(1,67)=0,84, p=0,362$, pero sí lo fue respecto del tiempo dedicado a trabajo matemático $\mathrm{NRP}, F(1,67)=4,3, p=0,041, \eta_{\mathrm{p}}{ }^{2}=0,06$. Controlando por las covariantes, aquellos profesores que hacen clases en cursos más bajos tienden a dedicar más tiempo a trabajo matemático NRP, en contraste con aquellos de cursos más altos (ver los descriptivos y los intervalos de confianza para la media en la Tabla 2). El efecto de la evaluación docente sobre el tiempo dedicado a pensamiento mecánico no resultó significativo, $F(1,67)=3,4, p=0,070$, al igual que para NRP, $F(1,67)=2,2, p=0,143$. La interacción Curso $\mathrm{x}$ Evaluación Docente no resultó significativa para pensamiento mecánico, $\mathrm{F}(1,67)=0,72, p=$ 0,398 , y para NRP, $\mathrm{F}(1,67)=2,63, p=0,110$. Aun cuando la interacción no alcanzó niveles de significación, la inspección de las medias sugiere que en cursos más avanzados el tiempo dedicado a trabajo matemático NRP podría cambiar según el nivel de desempeño de los profesores (ver Figuras 1 y 2 ). 
Tabla 1

Estadísticos Descriptivos del Tipo de Pensamiento Matemático

\begin{tabular}{|c|c|c|c|c|c|}
\hline $\begin{array}{l}\text { Tipo de pensamiento } \\
\text { matemático }\end{array}$ & Cursos agrupados & $\begin{array}{l}\text { Resultados evaluación } \\
\text { docente agrupados }\end{array}$ & Promedio & $\begin{array}{l}\text { Desviación } \\
\text { estándar }\end{array}$ & $N$ \\
\hline \multirow{9}{*}{ Pensamiento mecánico } & \multirow{3}{*}{$5^{\circ}-6^{\circ}$} & Bajo Estándar & 643,86 & 323,99 & 7 \\
\hline & & Sobre Estándar & 992,97 & 381,67 & 34 \\
\hline & & Total & 933,37 & 391,94 & 41 \\
\hline & \multirow{3}{*}{$7^{\circ}-8^{\circ}$} & Bajo Estándar & 886,86 & 553,88 & 7 \\
\hline & & Sobre Estándar & 823,88 & 349,04 & 25 \\
\hline & & Total & 837,66 & 392,93 & 32 \\
\hline & \multirow{3}{*}{ Total } & Bajo Estándar & 765,36 & 453,80 & 14 \\
\hline & & Sobre Estándar & 921,32 & 374,69 & 59 \\
\hline & & Total & 891,41 & 392,56 & 73 \\
\hline \multirow{9}{*}{$\begin{array}{l}\text { Trabajo matemático sin } \\
\text { resolución de problemas }\end{array}$} & \multirow{3}{*}{$5^{\circ}-6^{\circ}$} & Bajo Estándar & 658,57 & 327,27 & 7 \\
\hline & & Sobre Estándar & 789,12 & 476,30 & 34 \\
\hline & & Total & 766,83 & 453,54 & 41 \\
\hline & \multirow{3}{*}{$7^{\circ}-8^{\circ}$} & Bajo Estándar & 433,71 & 453,40 & 7 \\
\hline & & Sobre Estándar & 673,64 & 361,22 & 25 \\
\hline & & Total & 621,16 & 388,53 & 32 \\
\hline & \multirow{3}{*}{ Total } & Bajo Estándar & 546,14 & 397,40 & 14 \\
\hline & & Sobre Estándar & 740,19 & 431,72 & 59 \\
\hline & & Total & 702,97 & 429,62 & 73 \\
\hline
\end{tabular}

Tabla 2

Promedio, Desviación Estándar e Intervalos de Confianza de las Diferencias por Curso del Tipo de Pensamiento

\begin{tabular}{lccccc}
\hline \multirow{2}{*}{$\begin{array}{c}\text { Variable } \\
\text { dependiente }\end{array}$} & $\begin{array}{c}\text { Cursos } \\
\text { agrupados }\end{array}$ & Promedio * & $\begin{array}{c}\text { Desviación } \\
\text { estándar }\end{array}$ & $\begin{array}{c}\text { Intervalo de confianza 95\% } \\
\text { mínimo }\end{array}$ & $\begin{array}{c}\text { Límite } \\
\text { máximo }\end{array}$ \\
\hline Pensamiento & $5^{\circ}-6^{\circ}$ & 881,52 & 31,51 & 818,64 & 944,41 \\
mecánico & $7^{\circ}-8^{\circ}$ & 840,05 & 32,26 & 775,67 & 904,44 \\
NRP & $5^{\circ}-6^{\circ}$ & 761,57 & 73,47 & 614,93 & 908,21 \\
& $7^{\circ}-8^{\circ}$ & 542,44 & 75,21 & 392,32 & 692,57 \\
\hline
\end{tabular}

* Las covariantes que aparecen en el modelo son evaluadas de acuerdo a los siguientes valores: Edad = 50,48; Duración Total de Pensamiento Relevante $=1024,11$. 


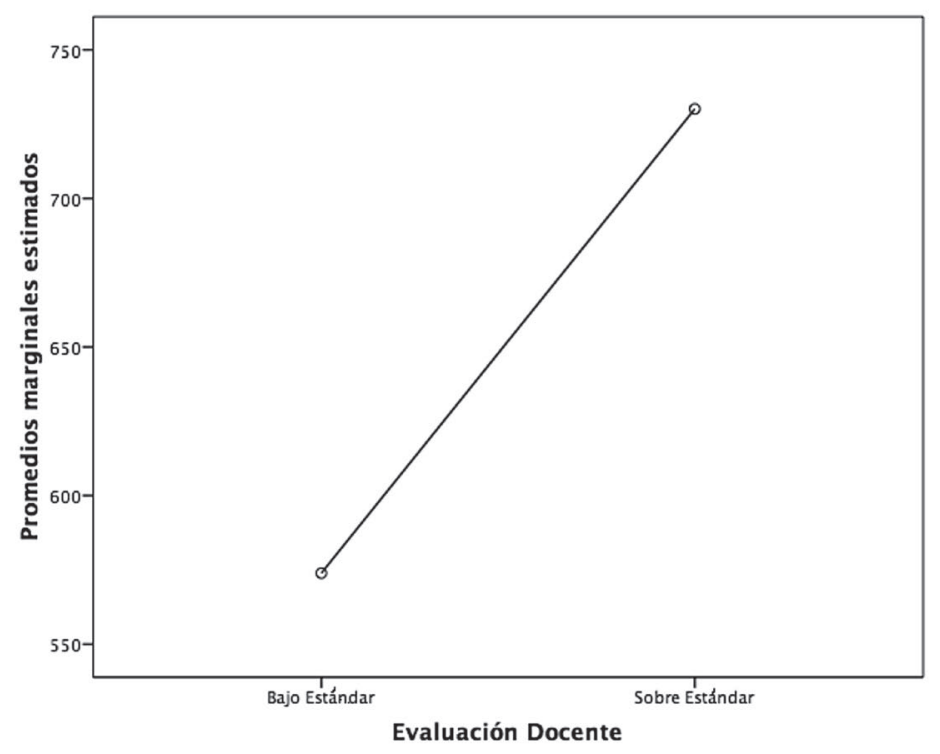

Figura 1. Promedios marginales estimados de trabajo matemático NRP según evaluación docente bajo estándar y sobre estándar. Las covariantes del modelo se estimaron en edad $=50,48$ años y duración total de pensamiento relevante $=1024,11$.

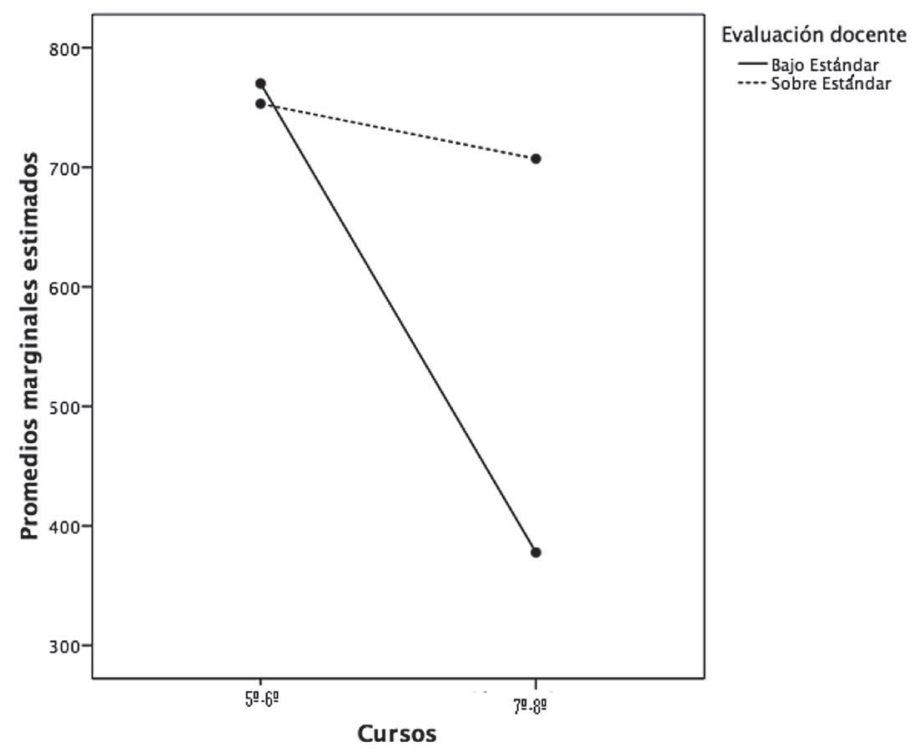

Figura 2. Promedios marginales estimados de trabajo matemático NRP según curso en el que ejerce el docente. Las covariantes del modelo se estimaron en edad = 50,48 años y duración total de pensamiento relevante $=$ 1024,11 . 


\section{Conclusiones}

Los resultados que obtuvimos en este estudio son compatibles con los hallazgos realizados en otros estudios sobre el aula matemática en Chile (Araya \& Dartnell, 2009; Preiss, 2010; Preiss et al., en prensa; Radovic \& Preiss, 2010) y sugieren que predomina la adherencia a una pedagogía externalista orientada a la práctica repetida de procedimientos. Tomados en el contexto de aquellos resultados acumulados previamente, permiten concebir el patrón adoptado por el discurso público en Chile y muestran que los profesores dedican un tiempo limitado de sus clases a pensar públicamente las matemáticas, es decir, a pensar cuestiones curriculares mediante discurso dirigido a la totalidad de los estudiantes. Cuando realizan RP, este tiempo está en su mayoría dedicado a entrenar destrezas y realizar procedimientos rutinarios, de manera que las nociones, más que desarrolladas, son comunicadas. En menor medida, los estudiantes se enfrentan a resolver problemas en los que tienen que movilizar conocimientos y conceptos y, cuando lo hacen, en la mayoría de los casos parecen no tener la oportunidad de cuestionar, fundamentar, vincular distintas estrategias y/o pensar acerca de la naturaleza de los objetos matemáticos relevantes para la tarea. Cuando decimos que tienen pocas oportunidades, nos referimos al hecho de que tienen poco acceso a un pensamiento público dedicado a resolver problemas y pocas oportunidades para pensar acerca de las operaciones que realizan y su naturaleza conceptual.

La inspección de otras variables no reveló diferencias relevantes: la inclusión de la edad o el desempeño obtenido en DM parecen no incidir en los aspectos fundamentales del patrón de discurso observado. En eso este estudio no se diferencia de otros realizados en Chile que también han encontrado una adherencia alta de los profesores a formas de enseñar que se revelan invariantes de acuerdo a variables demográficas o de desempeño (Preiss et al., en prensa). Una excepción es el impacto que el nivel del curso en que se enseña tiene sobre el tiempo dedicado a trabajo matemático NRP. Aquellos profesores que hacen clases en $5^{\circ}$ y $6^{\circ}$ básico tienden a dedicar más tiempo a trabajo matemático NRP, en contraste con aquellos que enseñan en $7^{\circ}$ y $8^{\circ}$ básico, los que tienden a involucrar más a sus alumnos en procesos de RP, aun cuando esta sea fundamentalmente de tipo mecánico. Adicionalmente, la inspección de las medias sugiere que el involucramiento de los estudiantes en procesos de RP en $7^{\circ}$ y $8^{\circ}$ es más intenso en aquellas clases de profesores cuyo desempeño ha sido identificado como bajo estándar. Desconocemos si este resultado es un artefacto producido por el formato de la evaluación que produce estos puntajes - por ejemplo, los evaluadores podrían estar castigando más a aquellas clases de los cursos avanzados que se focalizan más en RP, especialmente si esta es de tipo mecánico- o si responde a una tendencia real que se observa en profesores de distinto nivel de desempeño. Dado que no encontramos diferencias significativas en el tiempo dedicado a pensamiento mecánico entre profesores con diferentes niveles en su evaluación docente, la primera interpretación tiene algo de plausibilidad. Una de las limitaciones del estudio es que tanto el tamaño como la composición de los diversos grupos de la muestra no nos permiten asegurar nuestros resultados, especialmente aquellos referidos a las diferencias observadas según el nivel de desempeño de los profesores.

Es importante destacar que estos resultados deben ser interpretados tomando en consideración que la identificación de pensamiento razonado dista muchísimo del ideal de pensamiento con razonamiento. Raramente se observó argumentación o demostración. En la mayoría de los casos la codificación de pensamiento razonado obedeció a la vinculación de contenidos curriculares, específicamente, contenidos que están vinculados en el currículum propuesto por el Ministerio de Educación. En estos casos el tipo de pensamiento que aparece es muy discreto, tal como se puede observar en los ejemplos mostrados en el Anexo: mientras el ejemplo de resolución razonada de problemas ilustra un proceso de pensamiento que involucra o promueve razonamiento en la medida en que se habla de aquellas operaciones que llevaron a cabo los diferentes grupos para llegar al resultado (esto es, enfatizando las diferentes formas de llegar 
a un mismo resultado), el ejemplo de otro tipo de trabajo matemático razonado es un episodio expositivo de una profesora que presenta la información justificando y explicitando relaciones conceptuales.

Estos resultados tienen algunas implicancias relevantes para la formación docente. Muchas veces la formación docente a todo nivel está más centrada en entregar estrategias didácticas, tecnológicas y metodológicas, más que en promover el desarrollo de pensamiento matemático o una discusión acerca de su relevancia para la enseñanza. No se puede enseñar a pensar matemáticas sin poder pensarlas: el pensamiento se promueve ofreciendo oportunidades para pensar en conjunto. Esto requiere una guía experta y sostenida que tome en cuenta el valor de hablar matemáticamente, esto es, de hablar acerca de las operaciones y nociones propias de la disciplina, más allá de calcular. Estudiar el tipo de pensamiento que ocurre en la enseñanza nos ayuda a ver con claridad algo que mencionábamos en la introducción: que en sí misma una clase expositiva no es peor que una interactiva y que el trabajo grupal no es necesariamente mejor que la exposición docente. Todo depende de cómo se estimulen los procesos de pensamiento de los estudiantes. Este estudio provee categorías muy modestas para estudiar el pensamiento que ocurre en el discurso público en el aula matemática usando una muestra de tamaño medio. Continuar y complejizar su indagación es una tarea necesaria para informar más claramente la práctica pedagógica y diseñar actividades que orienten hacia la explicitación de procedimientos, conceptos y estrategias en la sala de clases. Si estas estrategias están ausentes, ellas debieran ser fomentadas y enseñadas explícitamente, tanto en la formación inicial como en actividades de perfeccionamiento a profesores en ejercicio.

\section{Referencias}

Alexander, R. (2000). Culture \& pedagogy: International comparisons in primary education. London, Reino Unido: Blackwell.

Alexander, R. J. (2004). Towards dialogic teaching: Rethinking classroom talk (2 $2^{\mathrm{a}}$ ed.). Cambridge, Reino Unido: Dialogos.

Alexander, R. J. (2008). Culture, dialogue and learning: Notes on an emergent pedagogy. En N. Mercer \& S. Hodgkinson (Eds.), Exploring talk in school (pp. 93-114). London, Reino Unido: Sage.

Araya, R. \& Dartnell, P. R. (2009). Saber pedagógico y conocimiento de la disciplina matemática en docentes de educación general básica y media. En Chile, Ministerio de Educación, Departamento de Estudios y Desarrollo (Ed.), Selección de investigaciones primer concurso FONIDE: evidencias para políticas públicas en educación (pp. 155-198). Santiago, Chile: Editor.

Asterhan, C. S. C. \& Schwarz, B. B. (2009). Argumentation and explanation in conceptual change: Indications from protocol analyses of peer-to-peer dialogue. Cognitive Science, 33, 374-400. doi:10.1111/j.1551-6709.2009.01017.x

Chamberlin, S. A. (2008). What is problem solving in the mathematics classroom? Philosophy of Mathematics Education Journal, 23, artículo 9. Extraído de http://people.exeter.ac.uk/PErnest/pome23/index.htm

Flanders, N. A. (1964). Interaction analysis in the classroom. Ann Arbor, MI: The University of Michigan.

Galton, M., Hargreaves, L., Comber, C., Wall, D. \& Pell, A. (1999). Inside the primary classroom: 20 years on. London, Reino Unido: Routledge.

Galton, M., Simon, B. \& Croll, P. (1980). Inside the primary classroom. London, Reino Unido: Routledge/Kegan Paul.

Galton, M. \& Williamson, J. (1992). Group work in the primary classroom. London, Reino Unido: Routledge.

Jansen, R. G., Wiertz, L. F., Meyer, E. S. \& Noldus, L. P. J. J. (2003). Reliability analysis of observational data: Problems, solutions, and software implementation. Behavior Research Methods, Instruments, \& Computers, 35, 391-399. doi:10.3758/BF03195516

Kieran, C., Forman, E. \& Sfard, A. (2001). Learning discourse: Sociocultural approaches to research in mathematics education [Guest Editorial]. Educational Studies in Mathematics, 46, 1-12. doi:10.1023/A:1014276102421

Lemke, J. L. (1990). Talking science: Language, learning, and values. Westport, CT: Ablex.

Mercer, N. \& Littleton, K. (2007). Dialogue and the development of children's thinking: A sociocultural approach. New York, NY: Routledge.

Milicic, N., Rosas, R., Scharager, J., García, M. R. \& Godoy, C. (2008). Diseño, construcción y evaluación de una pauta de observación de videos para evaluar calidad del desempeño docente. Psykhe, 17(2), 79-90. doi:10.4067/S071822282008000200007

Nunes, T. (1999). Mathematics learning as the socialization of the mind. Mind, Culture, and Activity, 6, 33-52. doi:10 .1080/10749039909524712

Nystrand, M., Gamoran, A., Kachur, R. \& Prendergast, C. (Eds.) (1997). Opening dialogue: Understanding the dynamics of language and learning in the English classroom. New York, NY: Teachers College Press.

Olson, D. R. (2003). Psychological theory and educational reform: How school remakes mind and society. Cambridge, MA: Cambridge University Press. 
Olson, D. R. (2005). Technology and intelligence in a literate society. En R. J. Sternberg \& D. D. Preiss (Eds.), Intelligence and technology: The impact of tools on the nature and development of human abilities (pp. 55-67). Mahwah, NJ: Lawrence Erlbaum.

Olson, D. R. (2009). Language, literacy and mind: The literacy hypothesis. Psykhe, 18(1), 3-9. doi:10.4067/S071822282009000100001

Preiss, D. D. (2009). The Chilean instructional pattern for the teaching of language: A video-survey study based on a national program for the assessment of teaching. Learning and Individual Differences, 19, 1-11. doi:10.1016/ j.lindif.2008.08.004

Preiss, D. D. (2010). Folk pedagogy and cultural markers in teaching: Three illustrations from Chile. En D. D. Preiss \& R. J. Sternberg (Eds.), Innovations in educational psychology: Perspectives on teaching, learning, and human development (pp. 325-355). New York, NY: Springer.

Preiss, D. D., San Martin, E., Alegría, I., Espinoza, A. M., Núñez, M. \& Ponce, L. (en prensa). Enseñar matemáticas fomentando la apropiación privada de términos y procedimientos: estructura y discurso en el aula matemática de primer ciclo. En Chile, Ministerio de Educación, Departamento de Estudios y Desarrollo (Ed.), Evidencias para políticas públicas en educación: selección de investigaciones Cuarto Concurso FONIDE. Santiago, Chile: Editor.

Radovic, D. \& Preiss, D. D. (2010). Patrones de discurso observados en el aula de matemática de segundo ciclo básico en Chile. Psykhe, 19(2), 65-79. doi:10.4067/S0718-22282010000200007

Rogoff, B., Matusov, E. \& White, C. (1996). Models of teaching and learning: Participation in a community of learners. En N. Torrance \& D. R. Olson (Eds.), The handbook of education and human development (pp. 388-414). Cambridge, MA: Blackwell.

Sfard, A. (2001). There is more to discourse than meets the ears: Looking at thinking as communicating to learn more about mathematical learning. Educational Studies in Mathematics, 46, 13-57. doi:10.1023/A:1014097416157

Steinberg, R., Empson, S. \& Carpenter, T. (2004). Inquiry into children's mathematical thinking as a means to teacher change. Journal of Mathematics Teacher Education, 7, 237-267. doi:10.1023/B:JMTE.0000033083.04005.d3

Stigler, J. W. \& Hiebert, J. (1999). The teaching gap: Best ideas from the world's teachers for improving education in the classroom. New York, NY: The Free Press.

Varas, M. L., Cubillos, L. F. \& Jiménez, D. (2009). Análisis de la calidad de clases de matemática: teorema de Pitágoras y razonamiento. En Chile, Ministerio de Educación, Departamento de Estudios y Desarrollo (Ed.), Selección de investigaciones primer concurso FONIDE: evidencias para políticas públicas en educación (pp. 123-153). Santiago, Chile: Editor.

Fecha de recepción: Junio de 2010.

Fecha de aceptación: Septiembre de 2011. 


\section{Anexo \\ Algunos Ejemplos de Códigos}

P: $\quad(\ldots)^{1}$ El dueño del negocio de allá de la esquina, como sabe que soy la profesora de matemática, vino ayer y me pidió que le hiciera una lista de precios. ¿Por qué? Porque la gente en esta época empieza a comprar más de una bebida. Compra cinco, compra ocho, entonces, a él se le complica estar sacando cuentas en ese momento, entonces me pidió que le hiciera una lista de precios ¿ya? y ustedes me van a ayudar a hacer esta lista de precios hoy día ¿ya? Se trata..., lo van a ver. A cada grupo le voy a pasar una guía para que me ayude a comprender esta lista de precios. Entonces, él me dio, el único dato que me dio que una bebida, una bebida coca cola salía setecientos pesos ¿ya? costaba setecientos pesos. Me van a ayudar a ver cuánto valen dos bebidas, cuánto valen tres bebidas y así le vamos a solucionar el problema a él para que no esté calculando rápidamente en el momento que esté vendiendo. Ya. Comencemos a trabajar en la guía, por favor.

P: (...) ¿estamos listos? (...) me ayudan a solucionar el problema. Ya. Vamos viendo, el grupo de ahí atrás, ¿solucionaron el problema? ¿Sacaron cuánto valen dos bebidas, tres bebidas, cuatro bebidas? Hasta cinco bebidas (individuales). Ya. ¿Cómo sacaron los datos?

Resolución razonada de problemas
A: Nosotras multiplicamos (...) una bebida vale setecientos.

P: Ya.

A: Entonces setecientos lo multiplicamos por dos, por tres, por cuatro, por cinco.

P: Ah, ya.

A: (...) resultado.

P: Perfecto y después le sacamos el resultado. El grupo de allá ¿cómo lo hicieron?

A: Nosotras sumamos (...) las dos bebidas.

P: Ya.

A: Lo dividimos por el resultado de las dos (...).

P: Fueron sumando, perfecto. Ustedes ¿qué hicieron?

A: Sacamos la constante de uno dividido (...).

P: Ah, ustedes lo sacaron por la constante. Perfecto. ¿Y ustedes?

A: (...) por dos, por tres, por cuatro, por cinco.

P: Ocuparon lo mismo que ustedes: multiplicaron. Ustedes también multiplicaron. Y ustedes ¿qué hicieron?

A: Nosotros sumamos.

P: ¿Sumaron? Ah, ya, perfecto. Nico (...). Está bien, está bien porque todos los grupos ocuparon distintas formas para poder sacarlo y vamos a ver ahora si sacaron los mismos resultados ¿cierto? porque unos sumaron, otros multiplicaron, otros usaron la constante, eso significa que dividieron. 


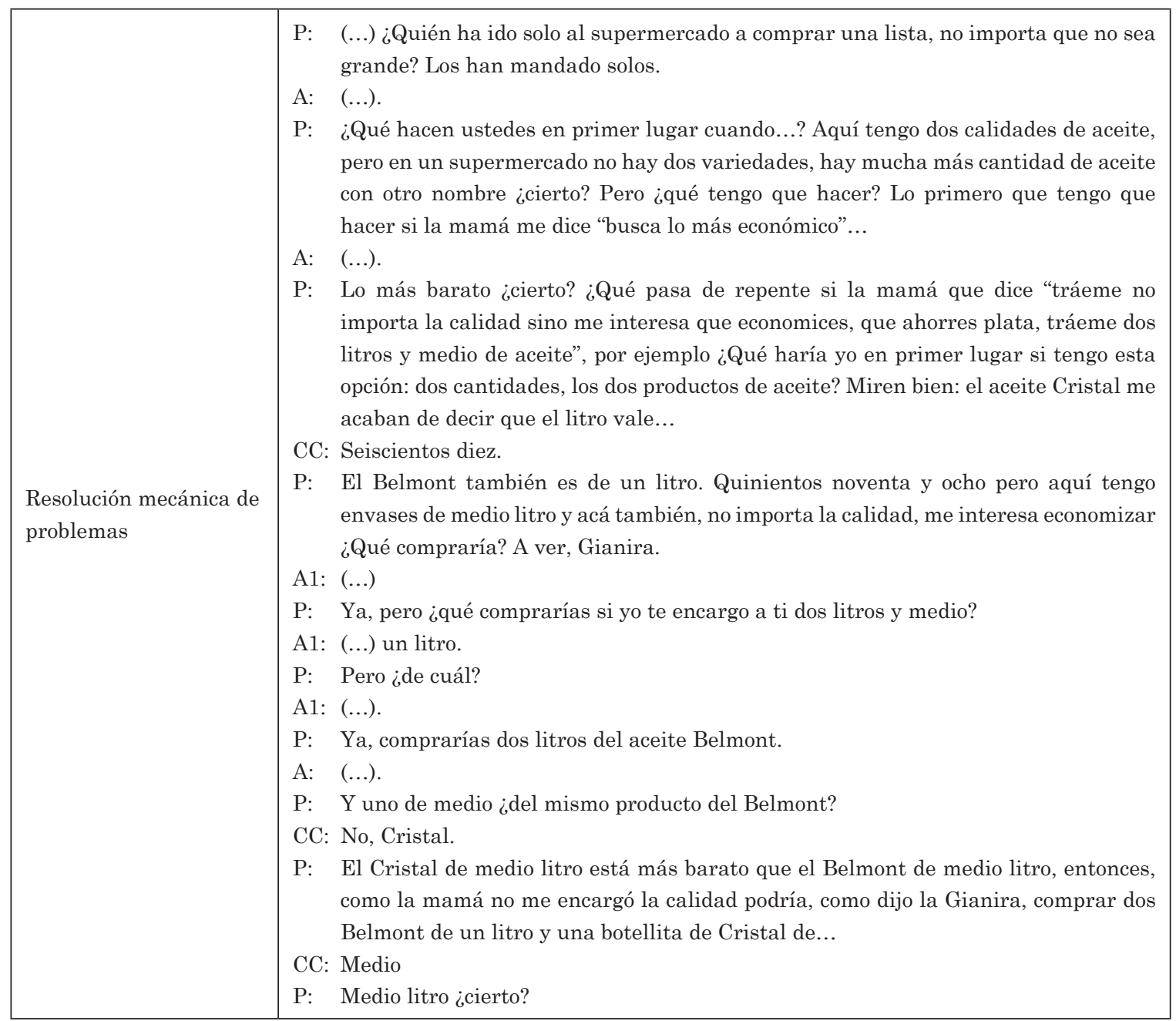

\begin{tabular}{|c|c|}
\hline $\begin{array}{l}\text { Trabajo matemático } \\
\text { mecánico }\end{array}$ & $\begin{array}{l}\text { P: Está bien, ya, vamos a ordenar ahora [los números] de mayor o menor. Ya, venga, } \\
\quad \text { Daniela. Ahora ordenamos de mayor o menor. Se supone que empezamos con los } \\
\text { CC: Positivos } \\
\text { P: Con los positivos (...) A ver ¿revisamos? Córrete un poquito para allá. Está el treinta } \\
\quad \text { y dos positivo ¿Bien? } \\
\text { CC: Sí. } \\
\text { P: Ya ¿el veintiuno positivo? } \\
\text { CC: Sí. } \\
\text { P: Ya ¿el quince positivo? } \\
\text { CC: Igual. } \\
\text { P: ¿El seis? } \\
\text { CC: Sí. } \\
\text { P: Ya ¿qué número vendría después? } \\
\text { CC: El cuatro. } \\
\text { P: ¿Después? } \\
\text { CC: El siete. } \\
\text { P: El siete y el... } \\
\text { CC: Ocho negativo } \\
\text { P: Ya, estamos bien, entonces. }\end{array}$ \\
\hline
\end{tabular}


P: Bien, el contenido que vamos a tratar hoy día es los polígonos ¿ya? Los polígonos son figuras geométricas que nacen de una línea poligonal. Una línea poligonal es un trazo ¿ya? Por ejemplo, si yo tomo este cuerpo. Toma ahí, por favor, toma tú acá, toma de acá, toma tú acá y toma tú acá. Ya, ahí lo que hace la cuerda es una línea poligonal ¿ya? que tiene un trazo, dos trazos, tres trazos...

A: Cuatro.

P: Cuatro trazos. Cada uno de sus compañeros está formando entre ellos un trazo y eso es una línea poligonal. Por favor ¿lo suelta [Javier]? Si ustedes ven acá en la pizarra, las trazadas en verde son líneas poligonales: tenemos esta, un trazo, dos, tres, cuatro, cinco trazos y la siguiente es una línea poligonal, tiene uno, dos, tres trazos. Las líneas poligonales siempre van a ser abiertas ¿ya? Es decir, no se intersectan, no se cruzan, son abiertas ¿ya? por eso se llaman líneas poligonales. Ahora, el polígono es una figura geométrica, polígono porque tiene varios lados, de ahí el origen del nombre: polígono, tiene varios lados, poli significa varios. Aquí tenemos una figura poligonal. Esta figura poligonal tiene tres trazos o segmentos. Esta figura poligonal tiene cuatro trazos o segmentos y esta figura poligonal tiene cuatro trazos o segmentos, es decir, se han cerrado. Un polígono se forma con líneas poligonales cerradas ¿ya? Por ejemplo, si yo ahora tomara... toma (...), por favor. Toma esta cara tú, por favor. Tómala tú, ahí, no la sueltes. Tómala tú (...), sigue allá, tómala tú. Tómala allá. Continúala a León. Continúala de León a Aguilera, de León a Aguilera. Ahora ¿si se paran un poquitito, por favor, ustedes? Se paran, se paran... levántenla arriba, ya, ahí hemos formado un polígono ¿por qué? Porque la línea poligonal que partió en Aguilera fue dando la vuelta, aquí habría un trazo o segmento, otro trazo o segmento, otro, otro, otro, otro, otro, otro y se cierra en Aguilera. Lo bajan, por favor. Suelten la punta y Aguilera la enrolla ¿ya? De ahí el origen de la figura geométrica. Para formar una figura geométrica... si yo, por ejemplo trazo, marco punto ¿cuántos puntos marqué ahí en la pizarra?

CC: Cuatro.

P: Cuatro puntos y esos puntos... coloco la regla acá, voy a decir aquí puntos colineales porque están en la misma línea. Estos puntos colineales los uno, ahí, uno este punto con este otro punto colineal. (...) Uno este otro punto, punto colineal. (...) Y vuelvo a decir ¿por qué se llaman colineales? Porque están en la misma línea. Aquí he formado una figura poligonal, formado una figura poligonal ¿ya? Entonces, tenemos que destacar nosotros que cuando una línea no se cierra se va a conocer con el nombre de línea poligonal. Cuando la línea se cierra se conoce como polígono.

El signo (...) indica fallas en el audio del video. 